\title{
ARE THERE POWDER DIFFRACTION DATA IN YOUR CLOSET?
}

Although there are over 70000 powder diffraction patterns in the 1994 Powder Diffraction File, PDF, there are many known phases for which there are no reported data. Because crystallography is one of the most important methods for the characterization of materials, many of the "missing" patterns may have been recorded in someone's diffraction laboratory. The goal of this editorial is to get everyone to make an effort to review the old laboratory files and reports and to recover these patterns for others to use. The data should be processed and screened to select the best available data set and then submitted to the International Centre for Diffraction Data, ICDD.

Every diffractionist who has been operating a characterization laboratory for ten years or more has had the experience of a user bringing in a new material for analysis. Many times the results of the analysis were prepared as a table of data which was then supplied to the requestor with a copy filed in the records of the laboratory. The ultimate distribution of the data at report time often is not at the discretion of the diffractionist, so the data may not have been incorporated in other than the file reports. Fortunately, some of these patterns ultimately find their way into the open literature as definitive information on the new phase. However, much of the data never makes it to the open literature, and thus is lost to the diffraction community.

The data in old files are still valuable for phase identification and characterization. It is particularly important to recover these data if there are no other powder data for the phase available for the diffraction community. It is also important if the data are an improvement on existing data. Some companies restrict the distribution of characterization data on new materials for proprietary reasons. Usually, there is a statute of limitations for such restrictions, and the data can be ultimately released for others to use if someone will take the initiative to process its release. Everyone reading this editorial should review the data in the old files, select useful data for processing, and initiate the procedures necessary to get such data released. In fact, some of the data might be the start for a paper for Powder Diffraction.

Although it is desirable to have powder data that meet the criteria for star, *, quality patterns in the PDF, it is not absolutely necessary to meet these criteria to be a very useful data set. In fact, low-resolution Debye-Scherrer-Gandolfi data may be the only data obtainable on a specific material because of the limited quantities of sample available. Completeness of the pattern and proper supporting documentation are more important than achieving ultimate accuracy in the numerical data as a criteria of usefulness of the information. If the data represent new phases or improvements on existing data and if the pattern can be shown to be representative of the single phase by indexing, optical, or chemical character- ization, the data should be submitted to the ICDD for PDF consideration. The ICDD recognizes submitters efforts and acknowledges individuals or companies with certificates and incentives. Do not submit data for uncharacterized samples nor the actual samples, as the ICDD has no facilities for recording the diffraction pattern.

Obviously, the best possible data should be selected for submission and the data should be supplimented by all other available characterization information on the phase. It does require some effort to prepare the data for submission, but it is well worth the time to assist in completing the coverage of phase information. The ideal form of presentation and type of supplimental information are described in a report of a Subcommittee of the American Crystallographic Association published as Calvert et al. (1979). This report may be consulted as a guide to preparing powder data, but it should not be considered a deterrent to submitting the data if all the desirable information is not available.

I doubt that there is a diffractionist who at one time or another has compared the data in the PDF with laboratory data and declared that the new experimental data are better than the reference data. Unfortunately, it is very rare that the diffractionist then submits these data to replace the reference pattern that was declared poor. Many laboratories have sets of reference data which are often consulted before going to the Powder Diffraction File. I have visited many laboratories that retain old Debye films because they act as good reference data. All these types of data are potential for review and submission.

For diffractionists at not-for-profit institutions, there is the Grant-in-Aid program of the ICDD. This program will provide small grants to cover the direct costs of preparing diffraction data for submission to the PDF. Grant proposals are considered once a year in December. Further information on this program can be obtained by contacting the ICDD.

It is time for everyone to check their closets, examine the old laboratory files, and activate the archived data into information useful to the diffraction community as a whole.

\section{References}

Calvert, L. D., Flippen-Anderson, J. L., Hubbard, C. R., Johnson, Q. C., Lenhart, P. G., Nichols, M. C., Parrish, W., Smith, D. K., Smith, G. S., Snyder, R. L., and Young, R. A. (1979). Accuracy in Powder Diffraction, edited by S. R. Block and C. R. Hubbard [National Bureau of Standards (now National Institutes for Standards and Technology), Gaithersburg, MD 20899, USA, Special Publication 567, issued February 1980.

Deane K. Smith Editor-in-Chief 